\title{
High Speed Titanium Coatings by Supersonic Laser Deposition
}

\author{
R. Lupoi, M. Sparkes, A. Cockburn and W. O’Neill \\ Institute for Manufacturing, Department of Engineering, University of Cambridge, \\ 17 Charles Babbage Road, CB3 0FS, Cambridge (UK). \\ Corresponding author is R. Lupoi \\ e-mail: r1372@cam.ac.uk \\ phone: (+44)01223764617 fax: (+44)01223464217
}

\begin{abstract}
The importance of metal coating technologies drives the continuous improvement of metal deposition techniques for application in a wide range of industrial sectors. This work presents the foundations of a new process technology for the deposition of titanium coatings on steel tube substrates using supersonic powder streams and impact site laser heating, known as Supersonic Laser Deposition (SLD). Metallic deposits are obtained under appropriate impact conditions without the need for exceeding the melting point of the deposited material or substrate leading to improved coating quality. Details of the experimental approach are presented along with the general characteristics of the titanium coating produced using this novel coatings method.
\end{abstract}

Key words: SLD, Cold Spray, Titanium coatings, Tubes, CFD, Laser deposition.

\section{Introduction}

Recent trends in the area of Rapid Prototyping (RP) and Manufacturing have led to the development of processes which provide end users with metal components which are not simply form and fit prototypes, but allow functional testing or direct implementation.

Technologies such as Laser Engineered Net Shaping (LENS), Direct Metal Deposition (DMD) and Selective Laser Melting (SLM) use a laser to melt and fuse metallic powder particles into a 
net or neat-net shape on a layer-by-layer basis. There are however disadvantages associated with these processes; they can suffer the detrimental effects of high temperature processing such as dilution between substrate and deposit, high thermally induced residual stresses, poor mechanical properties, unwanted microstructure / phase transformations and component distortion. There is also often the requirement of high purity inert environments to prevent oxidation during processing.

An increasing amount of effort is focusing on the development of a non-melting deposition process, known as Cold Spray (CS) [1,2,3]. In this method, powder is entrained within a supersonic gas jet that accelerates the particles up to high velocities before they impact the substrate. Upon impact particles undergo severe plastic deformation, which leads to bonding with a substrate and in the formation of a coating. Research studies carried out have produced valuable insights. It was suggested by Sakaki et al. [4] and demonstrated by Dykhuizen et al. [5] that an increase in particle temperature can lead to a boost in deposition efficiency and to a reduction in critical deposition velocity, as a result of particle softening. Increased gas temperatures heighten the risk of nozzle fouling when spraying low melting point metals such as aluminium [6]. Using a semi-empirical method, Assadi et al. [7] found that the critical velocity for a gas atomized copper powder increased with increasing yield stress and melting point, but decreased with higher impact temperature. Schimdt et al. [8] have provided more insights into the process, by demonstrating that particles of a larger size are characterized by a lower critical velocity.

However, there are inherent problems associated with the "cold" mechanism of deposition, which relies on a gas stream accelerating metallic powder particles to speeds approaching $1000 \mathrm{~m} / \mathrm{s}$. Drawbacks include the high operating costs associated with gas consumption and heating. The high flow rate requirement means the process can becomes exceptionally expensive when gases such as helium are necessary to deposit high strength materials. 
This paper presents experimental results for the coating of round steel substrates with titanium, using a novel coating technique currently under development at the University of Cambridge, known as Supersonic Laser Deposition (SLD). Preliminary studies were carried out by Bray et al. [9], which emphasised the vast potential of this coating technology. This process enables the coating fabrication with little or no melting, thus avoiding many of the problems that are associated with most laser based technologies. In SLD metallic powders are accelerated to velocities around half of those used in CS. A laser is used to heat the deposition zone in order to soften both the substrate and particles to allow the formation of a coating at a much reduced impact velocity.

\section{SLD process description}

A high pressure (0-30bar range) nitrogen gas supply is split and delivered to a convergingdiverging nozzle both directly and via a Praxair 1264HP high pressure powder feeder where metal powder particles are en-trained, as shown in Figure 1. The two streams recombine and pass through the nozzle where they are accelerated to supersonic speed. The high velocity, powderladen gas jet exits the nozzle and is directed towards a substrate. The powder stream impacts a region of the substrate that is simultaneously illuminated by a YLS fibre laser (IPG), with a maximum power of $4 \mathrm{KW}$. The deposition zone temperature is monitored by a high speed IRPyrometer.

The supersonic nozzle, laser head and pyrometer are arranged on a single mount, while the substrate can be moved using a CNC X-Y system. A spindle is also incorporated in the arrangement and shown in Figure 2, to allow for the processing of cylindrical tubes. Pressure regulators and flow valves to ensure the required operating conditions are fully computer controlled. 
The SLD process evolves from an initial system, which is described by Bray et al. [9] and known as Laser assisted Cold Spray (LCS). In this preliminary set up a 1KW diode laser was used, and the working chamber was designed for the fabrication of single tracks or flat coatings only. Experimental observations have suggested the use of a different laser type combined with the capability of a higher maximum power may considerably improve the process.

\section{Nozzle design}

The nozzle geometry is De-Laval type with a linear converging-diverging internal profile (circular cross-section), characterized by a throat of $2 \mathrm{~mm}$ diameter and an overall length of 210mm. The subsonic and supersonic internal profile contours were determined by a Computational Fluid Dynamic (CFD) analysis carried out with Fluent v6.3.26; Figure 3 shows the velocity field in the nozzle outlet zone. Typically, the Standoff Distance between the exit of the nozzle and substrate is set to values within the range $40 \mathrm{~mm}$ to $60 \mathrm{~mm}$. When spraying with $30 \mathrm{bar}$ inlet pressure at room temperature, the gas exit velocity approaches $680 \mathrm{~m} / \mathrm{s}$ as the figure shows; on the other hand particles speed increases throughout the channel up to a maximum level, dependent on the feedstock material and size. A spherical titanium particle $(30 \mu \mathrm{m}$ diameter) is predicted to reach the speed of $474 \mathrm{~m} / \mathrm{s}$ at impact, considerably lower than its critical velocity for CS $[8,10]$ only achievable with gas-heating or when using helium as carrier gas.

\section{Titanium coatings on steel tubes}

Being a solid-state coating technology (since melting temperatures are not crossed), SLD has the potential of a relatively high processing speed and controllability level. Figure 4 shows a close up picture of a $3 \mathrm{~mm}$ thick titanium coating over a $50 \mathrm{~mm}$ diameter low carbon steel tube (conforming to BS 6323-part 4). The coating was produced with 30bar nozzle inlet pressure at room temperature. The initial generated thickness of the coating was $4 \mathrm{~mm}$, for a total processing time of approximately 3 minutes; however it was turned on a lathe to demonstrate properties 
such as machinability and ductility. No cracks or coating detachment were observed in this operation. The powder used was CP titanium ( $-45 \mu \mathrm{m}$ spherical, grade 2$)$. The required final thickness was achieved by the deposition of overlapped "high-speed" thin coatings, therefore by following a multi-layer building strategy. Adhesion strength results with the substrate (pull-off test) have given an average value in the excess of $77 \mathrm{MPa}$, almost four times higher than the achievable level with conventional CS [11].

A micrograph cross-section of a $0.2 \mathrm{~mm}$ thick turned titanium coating by SLD on a steel tube is shown in Figure 5. The deposit appears well compacted with a low level of porosity. Microhardness measurements on the external coating surface have revealed an average value of $272 \mathrm{HV}_{0.3}$, approximately 1.5 times higher than conventional pure titanium grade 2 . The coating is therefore shown to be characterized by enhanced properties. A detailed analysis is currently under investigation.

\section{Conclusions}

An alternative coating technology, Supersonic Laser Deposition (SLD), has been introduced. This technique incorporates the supersonic metal particle stream found in Cold Spray, with laser heating of the deposition zone. CFD analysis was used to design the internal contour of the nozzle. It was observed that laser heating of the processing site did enable the deposition of highvelocity particles, which would otherwise rebound. SLD was successfully applied for the formation of high speed titanium coatings over $50 \mathrm{~mm}$ diameter steel tubes. The final coating thickness of $4 \mathrm{~mm}$ was achieved in approximately 3 minutes processing time. Adhesion measurements gave a bond strength in the excess of $77 \mathrm{MPa}$. In addition micrograph crosssections have shown a high level of compaction and low porosity, for an average hardness of $272 \mathrm{HV}_{0.3}$ on the coating external layer. 


\section{Acknowledgements}

The authors wish to express their gratitude to IPG photonics for the valuable technical support, and to Ms. Fang Luo for providing expertise in samples preparation and analysis.

\section{References}

[1] Pattison, J., et al., Cold gas dynamic manufacturing: A non-thermal approach to freeform fabrication. International Journal of Machine Tools and Manufacture, 2007. 47(3-4): p. 627-634.

[2] Champagne, V., USA, The cold spray materials deposition process: Fundamentals and applications. 2007: Woodhead Publishing Limited.

[3] Morgan, R., et al. Analysis of cold gas dynamically sprayed aluminium deposits, Materials Letters, 58 (2004) 1317-1320.

[4] Sakaki, K. and Y. Shimizu, Effect of increase in entrance convergent section length of gun nozzle on HVOF and its application to CGDS nozzle design. Journal of Thermal Spray Technology., 2001. 10(3): p. 487-496.

[5] Dykhuizen, R.C. and R.A. Neiser. Optimising the cold spray process. in ITSC. 2003: ASM International.

[6] Stoltenhoff, T., et al. Optimisation of the cold spray process. in ITSC. 2001: ASM International.

[7] Assadi, H., et al., Bonding mechanism in cold gas spraying. Acta Materialia, 2003. 51(15): p. 4379-4394.

[8] Schmidt, T. et al. From Particle Acceleration to Impact and Bonding in Cold Spraying, Journal of Thermal Spray Technology, 18(5-6), Mid-December 2009, 794-808.

[9] Bray, M. et al., The laser assisted cold spray process and deposit characterization, Surface \& Coatings Technology, 203 (2009), 2851-2857.

[10] Schmidt, T., et al., Development of a generalised parameter window for cold spray deposition. Acta Materialia, 2006. 54: p. 729-724.

[11] Marrocco, T., Production of Titanium Deposits by Cold-Gas Dynamic Spray: Numerical Modeling and Experimental Characterization, Journal of Thermal Spray Technology, Volume 15(2) June 2006. 


\section{List of Figures}

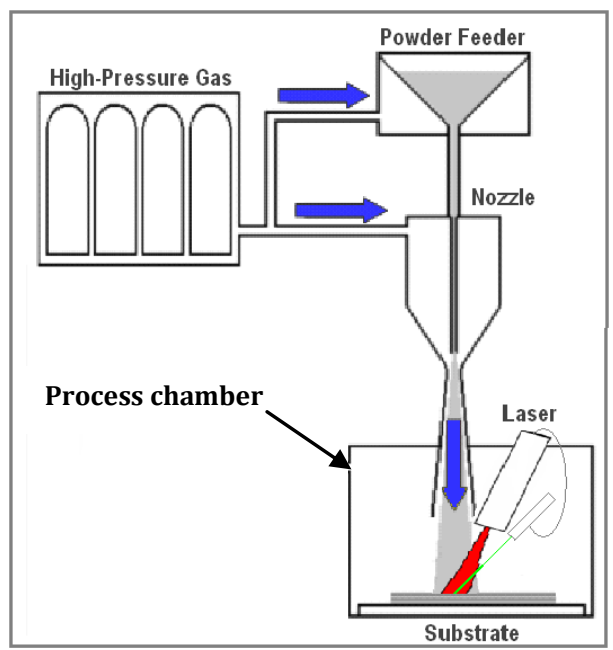

Figure 1: SLD schematics.

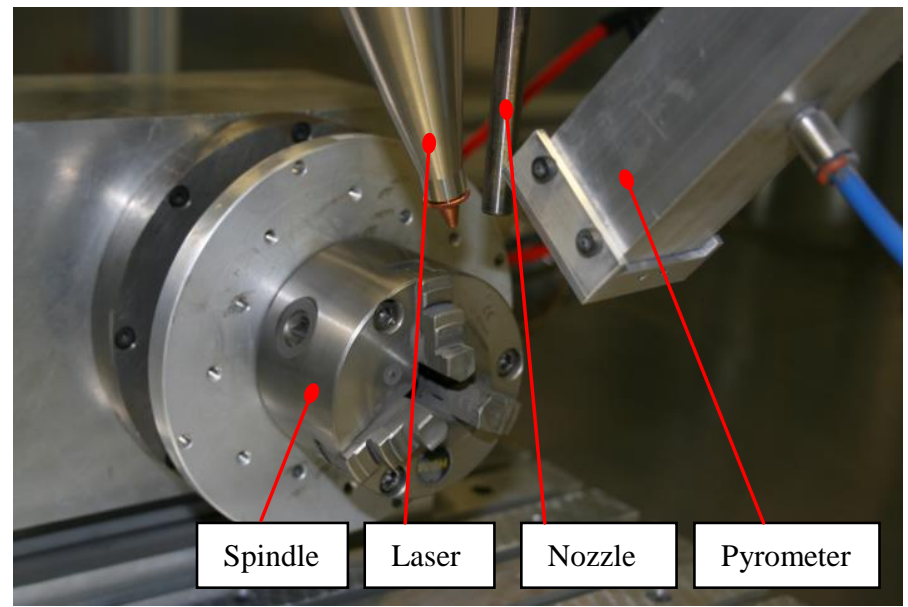

Figure 2: Close-up picture of main components.

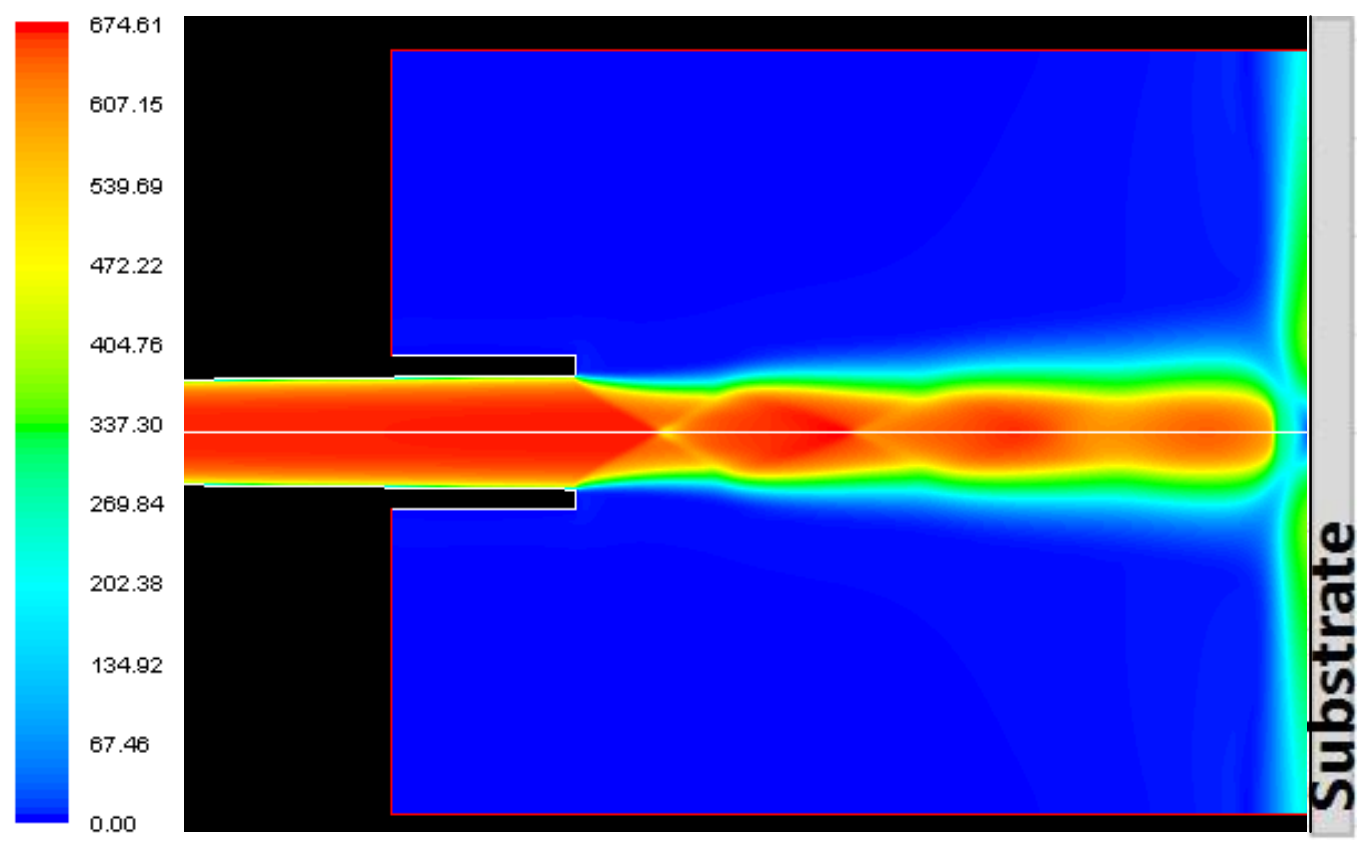

Figure 3: Nitrogen velocity magnitude $(\mathrm{m} / \mathrm{s})$ at nozzle exit and substrate impact by a CFD analysis. 


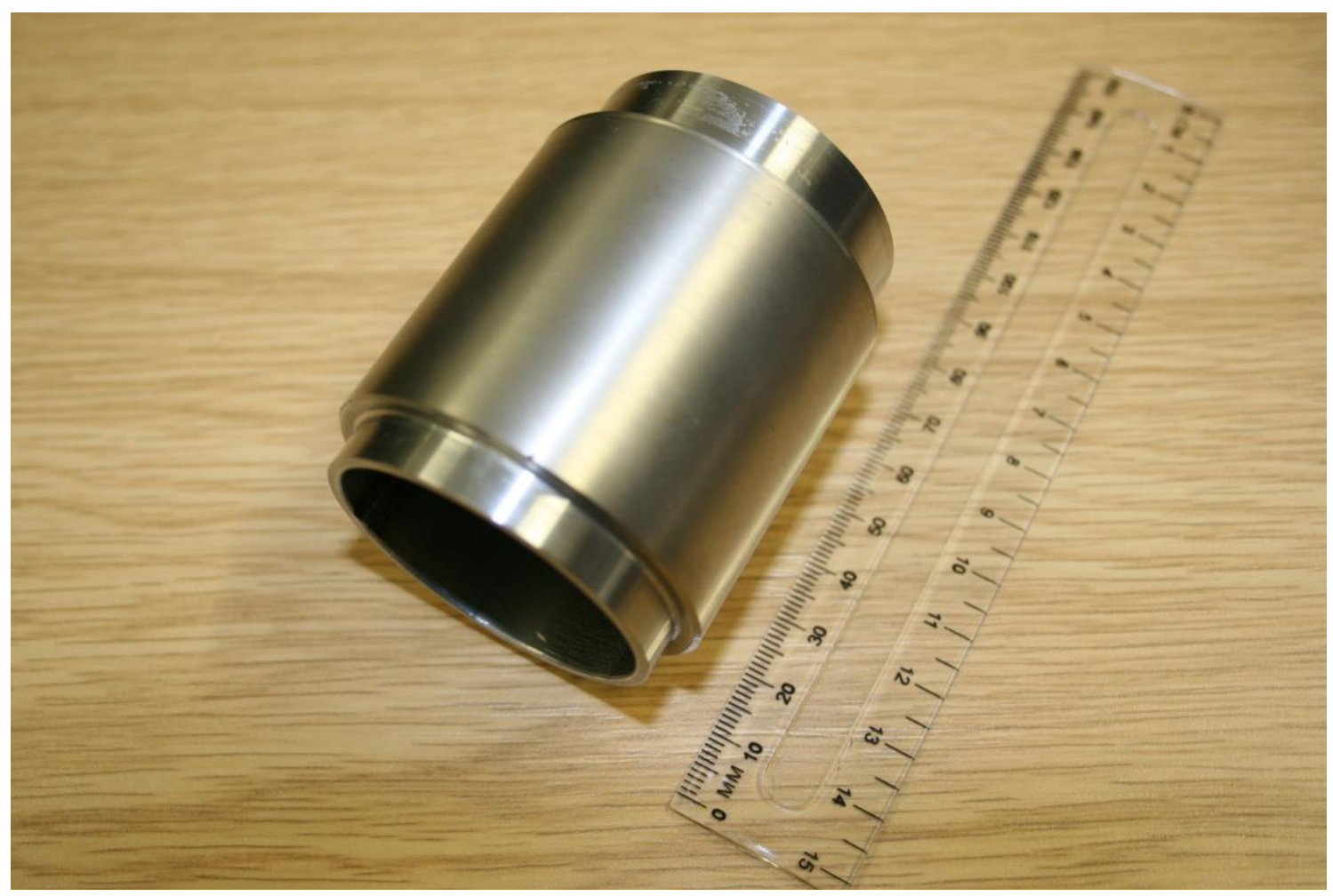

Figure 4: Thick titanium coating ( $3 \mathrm{~mm}$ ) on a steel tube by SLD.

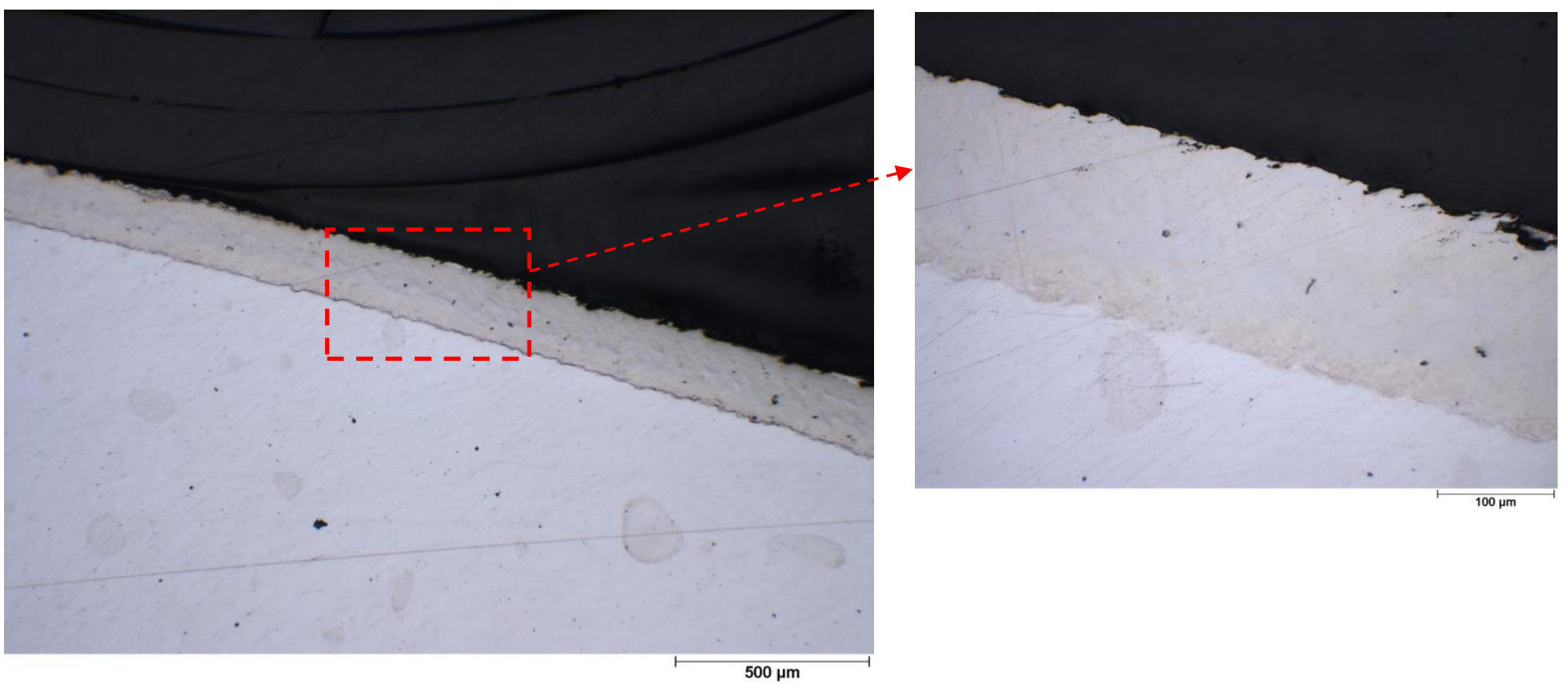

Figure 5: Titanium coating micrograph cross-section by SLD. 